\title{
Is essential fatty acid interconversion an important source of PUFA in humans?
}

\author{
Graham C. Burdge* \\ Human Health and Development, Faculty of Medicine, University of Southampton, Southampton SO16 6YD, UK \\ (Submitted 19 September 2018 - Final revision received 28 November 2018 - Accepted 7 December 2018 - First published online 28 February 2019)
}

\section{Abstract}

Humans can obtain pre-formed long-chain PUFA from the diet and are also able to convert essential fatty acids (EFA) to longer-chain PUFA. The metabolic pathway responsible for EFA interconversion involves alternating desaturation and carbon chain elongation reactions, and carbon chain shortening by peroxisomal $\beta$-oxidation. Studies using stable isotope tracers or diets supplemented with EFA show that capacity for PUFA synthesis is limited in humans, such that DHA (22:6n-3) synthesis in men is negligible. PUFA synthesis is higher in women of reproductive age compared with men. However, the magnitude of the contribution of hepatic PUFA synthesis to whole-body PUFA status remains unclear. A number of extra-hepatic tissues have been shown to synthesise PUFA or to express genes for enzymes involved in this pathway. The precise function of extra-hepatic PUFA synthesis is largely unknown, although in T lymphocytes PUFA synthesis is involved in the regulation of cell activation and proliferation. Local PUFA synthesis may also be important for spermatogenesis and fertility. One possible role of extra-hepatic PUFA synthesis is that it may provide PUFA in a timely manner to facilitate specific cell functions. If so, this may suggest novel insights into the effect of dietary PUFA and/or polymorphisms in genes involved in PUFA synthesis on health and tissue function.

\section{Key words: Desaturase: Elongase: Fatty acid desaturase: Liver: Lymphocytes: Fatty acids}

PUFA are important components of cell membranes. Variations in the proportions of individual PUFA can alter the cell function by modulating the fluidity of the phospholipid bilayer, thus influencing the activity of integral membrane proteins ${ }^{(1)}$. PUFA can also act as substrates for cell signalling processes. Changes in the relative proportions of individual PUFA may modify cell function by changing the nature of lipid second messengers, including eicosanoids ${ }^{(2)}$, diacylglycerol and phosphatidic $\operatorname{acid}^{(3,4)}$. For example, activated macrophages produce 2-series PG from arachidonic acid (20:4n-6) through the activity of cyclo-oxygenase but synthesise less biologically potent 3-series PG from EPA $(20: 5 n-3)^{(2)}$. Protein kinase $C_{\alpha}$ has been shown to be preferentially activated by 1,2-dipalmitoyldiacylglycerol (DPD) compared with 1-stearoyl, 2-docosahexaenoyldiacylglycerol (SDD). However, activation of protein kinase $\mathrm{C}_{\gamma}$ by SDD is greater compared with DPD ${ }^{(5)}$. PUFA can modify cell function at the level of gene transcription by acting as ligands for $\operatorname{PPAR}^{(6)}$, and recent findings suggest that PUFA can modify epigenetic processes ${ }^{(7)}$. Thus, maintenance of the phospholipid composition of cell membranes and, consequently, normal cell activity requires an adequate, timely supply of specific PUFA. These can be obtained pre-formed from the diet via the bloodstream. However, this may be a precarious strategy for maintaining tissue function because dietary choice and temporal variation in intake may limit the capacity of the diet to supply individual PUFA against changing demands; for example, during an immune response to infection.
Mammals can convert the essential fatty acids (EFA) linoleic acid (18:2n-6) and $\alpha$-linolenic acid (18:3n-3) to longer-chain, more unsaturated fatty acids that are required for cell function. The purpose of this review is to assess whether hepatic and extra-hepatic PUFA syntheses are important for meeting demands for PUFA, primarily in humans.

\section{Hepatic PUFA synthesis in mammals other than humans}

Mammalian cell membranes contain C18, C20 and C22 PUFA, which are classified as $n-6, n-3$ or $n-9$ depending on the position of the first double bond from the methyl end of the hydrocarbon chain. The MUFA oleic acid (18:1n-9) can be synthesised de novo from non-lipid precursors by the activity of the fatty acid synthase complex followed by insertion of a double bond at $\Delta 9$ position by stearoyl-CoA desaturase ${ }^{(8)}$. During dietary EFA deficiency, 18:1n-9 can be converted to Mead acid $(20: 3 n-3)$ by the sequential activities of $\Delta 6$ desaturase, elongase 5 and $\Delta 5$ desaturase $^{(8)}$ (Fig. 1). EFA deficiency and $20: 3 n-9$ synthesis are rare in humans and occur primarily in patients receiving artificial nutrition ${ }^{(9)}$. However, the precise biological role of conversion of $18: 1 n-9$ to $20: 3 n-9$ is not known. One possibility is that $20: 3 n-9$ may, at least in part, compensate for decreased membrane fluidity due to reduced availability of PUFA for incorporation into the phospholipid bilayer. Alternatively, synthesis of $20: 3 n-9$ involves, in part,

Abbreviations: EFA, essential fatty acid; FADS, fatty acid desaturase; PBMC, peripheral blood mononuclear cells; PC, phosphatidylcholine.

* Corresponding author: Professor G. C. Burdge, email g.c.burdge@soton.ac.uk 


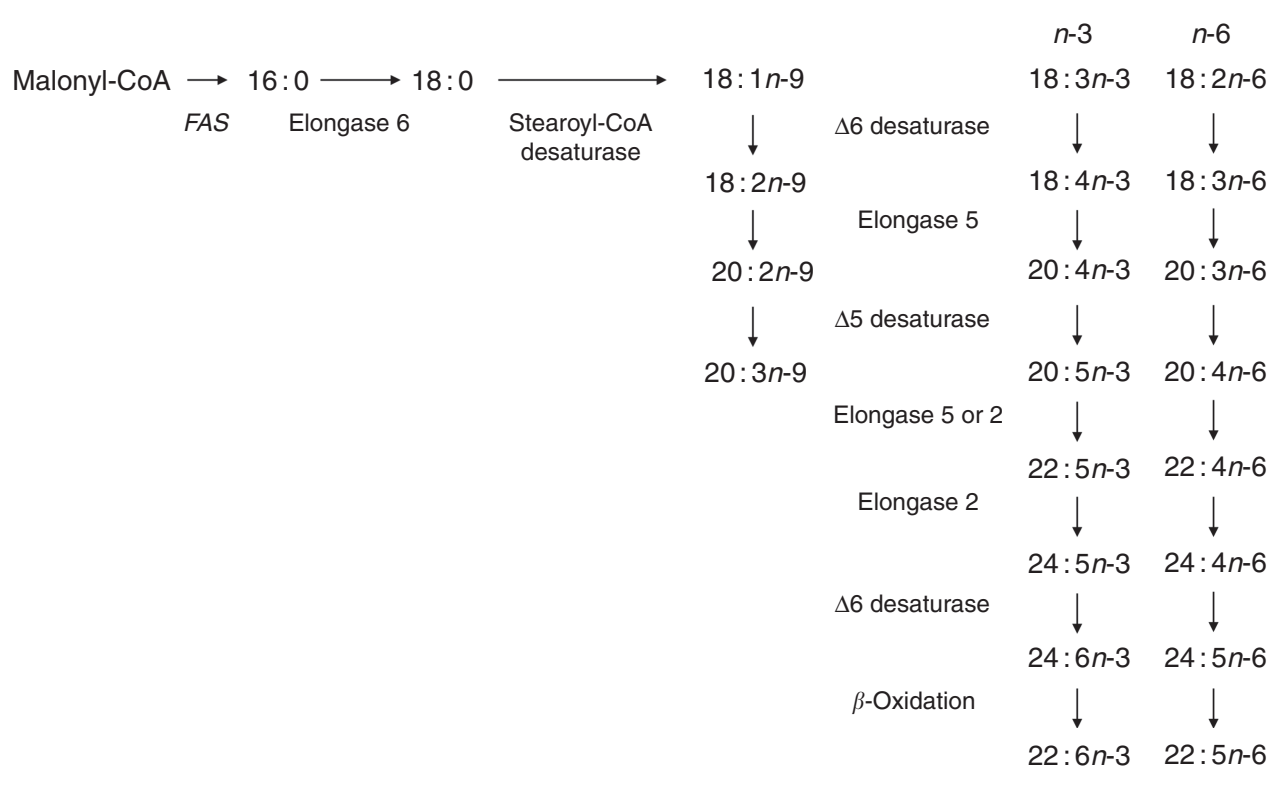

Fig. 1. The pathway for synthesis of longer-chain $n-9$ fatty acids overlaps in part with metabolic reactions involved in $n-3$ and $n-6$ PUFA biosynthesis. FAS, fatty acid synthase.

enzymes that catalyse PUFA synthesis (Fig. 1) and so conversion of $18: 1 n-9$ to $20: 3 n-9$ may be an artefact of reduced flux of EFA through the PUFA synthesis pathway.

Mammals do not express $\Delta 12$ or $\Delta 15$ desaturases which catalyse the conversion of $18: 1 n-9$ to $18: 2 n-6$ and $18: 2 n-6$ to $18: 3 n-3$, respectively. Consequently mammals are dependent upon either consumption of pre-formed $\geq 20$ carbon PUFA in their diet or conversion of $18: 2 n-6$ and $18: 3 n-3$ to longerchain, more unsaturated fatty acids to meet their PUFA requirements. The general pathway for conversion of $18: 2 n-6$ and 18:3n-3 to longer-chain, more unsaturated PUFA was elucidated in the $1960 \mathrm{~s}$ by studies carried out using rodent liver $^{(10,11)}$ (Fig. 1). The first desaturation reaction, catalysed by $\Delta 6$ desaturase, is followed by malonyl-CoA-dependent carbon chain elongation by elongase 5 and then $\Delta 5$ desaturation to form 20:4n-6 and 20:5n-3 from $18: 2 n-6$ and 18:3n-3, respectively (Fig. 1); $20: 4 n-6$ can be converted to $22: 4 n-6$ and $20: 5 n-3$ to $22: 5 n-3$ by the addition of two carbons by either elongase 5 or elongase 2 (Fig. 1). Although the first reaction catalysed by $\Delta 6$ desaturase is generally assumed to be rate limiting, the chain elongation reactions that follow the formation of $22: 5 n-3$ and $22: 4 n-6$ have also been proposed as the metabolic control points ${ }^{(12)}$. Synthesis of $22: 5 n-6$ and $22: 6 n-3$ was originally proposed to involve $\Delta 4$ desaturation ${ }^{(13)}$. However, this view was superseded because of the apparent absence in rat liver microsomes of an enzyme with $\Delta 4$ desaturase activity and the demonstration that synthesis of $22: 5 n-6$ and $22: 6 n-3$ involves further chain elongation by elongase 2 to form the intermediates $24: 4 n-6$ and $24: 5 n-3$, followed by $\Delta 6$ desaturation and subsequent translocation of the products, $24: 5 n-6$ and $24: 6 n-3$, to peroxisomes. $22: 5 n-6$ and $22: 6 n-3$ are formed by the removal of two carbons by single cycle of $\beta$-oxidation ${ }^{(14-16)}$. However, Park et al. ${ }^{(17)}$ have shown that the MCF7 human breast cancer cell line, which does not express $\Delta 6$ desaturase activity, expresses $\Delta 4$ desaturase activity when transfected with fatty acid desaturase (FADS) 2 and FADS1, which encodes $\Delta 6$ and $\Delta 5$ desaturases, respectively. However, $\Delta 4$ desaturase activity remains to be demonstrated in wild-type, untransformed primary cells.

\section{Hepatic PUFA biosynthesis in humans}

In marked contrast to rodents, humans have limited capacity for conversion of EFA to longer-chain PUFA ${ }^{(18,19)}$. Studies using stable isotope tracer technology to assess whole body, in essence hepatic, interconversion of $18: 3 n-3$ in men or mixed groups of men and women have shown consistently that conversion to $20: 5 n-3$ is limited to $<10 \%$ of the administered labelled $18: 3 n-3$, and that only trace amounts of $22: 6 n-3$ are formed $(<0.05 \% \text { of ingested } 18: 3 n-3)^{(18-20)}$. Moreover, conversion of $\left[{ }^{13} \mathrm{C}\right] 18: 3 n-3$ to $22: 6 n-3$ has been shown to be independent of relative intakes of $18: 3 n-3$ and $18: 2 n-6^{(20)}$. Thus, the contribution of PUFA synthesis to $22: 6 n-3$ formation appears to be negligible, although one study in men estimated conversion of $18: 3 n-3$ to $22: 6 n-3$ to be approximately $4 \%$ of the administered dose ${ }^{(21)}$. It is not known why $22: 6 n-3$ synthesis was greater in this study compared with others. Conversion of $\left[{ }^{13} \mathrm{C}\right] 18: 2 n-6$ to $20: 4 n-6$ in men has also been shown to be extremely limited (approximately $<0.2 \%$ of ingested labelled fatty acid) ${ }^{(20)}$.

There are major challenges in interpreting the concentrations of stable isotope-labelled fatty acids in blood in terms of EFA interconversion, which have been reviewed elsewhere ${ }^{(19)}$. However, such estimates are consistent with the effect of increased 18:3n-3 intake (between 4 and $20 \mathrm{~g} / \mathrm{d}$ ) on the proportions of $20: 5 n-3,22: 5 n-3$ and $22: 6 n-3$ in blood and cell lipids in men or in mixed groups of men and postmenopausal women ${ }^{(22,23)}$. These studies showed that greater 18:3n-3 intake was associated with increased proportions of $20: 5 n-3$ and 
$22: 5 n-3$. Based on data summarised in Burdge \& Calder ${ }^{(23)}$, $18: 3 n-3$ intake predicted $66 \%$ of the variation in the proportion of $20: 5 n-3$ in plasma phospholipids such that doubling 18:3n-3 intake approximately doubled the change in $20: 5 n-3$ status. However, there was no significant increase in the proportion of $22: 6 n-3$ and in four of nine studies $22: 6 n-3$ status decreased during $18: 3 n-3$ supplementation to below the baseline level ${ }^{(23)}$. In addition, dietary supplementation with $18: 3 n-3,18: 4 n-3$ or $20: 5 n-3$ increased the proportions of $20: 5 n-3$ and $22: 5 n-3$ in blood but was accompanied by a statistically non-significant decrease in $22: 6 n-3$ status ${ }^{(24)}$. One possible explanation for the reduction in $22: 6 n-3$ status is that the rate of $22: 6 n-3$ synthesis was less than the rate of turnover.

Women and females of other species appear to have greater capacity for synthesis and higher 22:6n-3 status than their male counterparts. Conversion of $\left[{ }^{13} \mathrm{C}\right] 18: 3 n-3$ to $20: 5 n-3$ in women of reproductive age has been estimated to be $21 \%$ (compared with $<10 \%$ in men) and conversion to $22: 6 n-3$, $9 \%$ (compared with $<0 \cdot 05 \%$ in men $)^{(25,26)}$. This observation is supported by the findings of a systematic review of fifty-one observational studies that showed the proportions of $20: 4 n-6$ and $22: 6 n-3$ in blood phospholipids were typically $20 \%$ higher in women than in men ${ }^{(27)}$ and also by a recent kinetic modelling study in overweight women ${ }^{(28)}$. Female rodents ${ }^{(29,30)}$ and great tits (Parus major) ${ }^{(31)}$ also have higher 22:6n-3 status compared with males, which suggests this difference in PUFA status and metabolism between sexes may have been conserved during evolution. Oestrogen is an agonist for PUFA synthesis ${ }^{(25,32)}$ and hence may be responsible for greater PUFA synthesis in females than in males. However, studies in postmenopausal women, in primary human hepatocytes and in HepG2 hepatocarcinoma cells, suggest that progesterone may also be involved $(33,34)$

Dietary supplementation with $9.5 \mathrm{~g} / \mathrm{d} 18: 3 n-3$ induced a greater increase in the proportion of $20: 5 n-3$ in women (age 53.5 years) compared with men (age 50.5 years), but there was no difference between sexes in the proportion of $22: 6 n-3$ at the end of the study ${ }^{(35)}$. Furthermore, the change in plasma $20: 5 n-3$ status was associated negatively with age in women but not in men ${ }^{(35)}$. These findings suggest that, at least in women of post-reproductive age, the contribution of PUFA biosynthesis to plasma PUFA status is similar to men. Whether this pathway is more important for meeting the demands for PUFA of younger women has yet to be determined.

\section{Hepatic PUFA biosynthesis in pregnancy}

Pregnancy involves increased demands on the mother for PUFA, to meet both her requirements and those of her fetus. The human fetus accumulates 22:6n-3, beginning in the second trimester, particularly into the central nervous system ${ }^{(13,36)}$ and deficits in 22:6n-3 incorporation into developing brain have been associated with cognitive deficits in non-human primates $^{(37)}$. The concentration of $22: 6 n-3$, but not $20: 4 n-6$, has been shown to increase in plasma phosphatidylcholine (PC) during the second trimester of pregnancy, specifically an increase in PC16:0/22:6 ${ }^{(38)}$, which precedes the period of prenatal 22:6n-3 accumulation into fetal brain ${ }^{(36)}$. One interpretation is that such adaptation of maternal liver lipid metabolism may facilitate adequate supply of $22: 6 n-3$ to the fetal brain ${ }^{(39)}$. Total plasma $22: 6 n-3$ concentration has also been shown to increase between 18 and $29 \mathrm{~d}$ post-conception in women undergoing assisted reproduction ${ }^{(40)}$. This change in plasma PC composition has been shown in rats to involve changes in the composition of the maternal hepatic diacylglycerol pool destined for PC synthesis and decreased acyl remodelling of $s n-1$ 16:0 to $s n-1$ 18:0 PC molecular species ${ }^{(41)}$ and to be accompanied by increased liver Fads1 and Fads2 mRNA expression ${ }^{(29,42)}$, possibly by the action of progesterone $^{(42)}$.

Despite evidence of coordinated changes in maternal hepatic PUFA metabolism, the contribution, if any, of such adaptations to meeting maternal and fetal demands for PUFA is not known. In pregnant women, maternal plasma PC $22: 6 n-3$ concentration tracks during gestation such that the level in early pregnancy (11-17 weeks) predicts $21 \%$ of the variation in late pregnancy $(35 \text { weeks })^{(43)}$. Therefore, it may be expected that position in the rank order of maternal $22: 6 n-3$ status, which is a proxy for $22: 6 n-3$ availability to the developing fetus, would influence the cognitive function of the child. However, maternal plasma $22: 6 n-3$ concentration during pregnancy has not been associated with intelligence quotient (IQ) or markers of executive cognitive function at ages 4 or $6-7$ years ${ }^{(43,44)}$, cognition at 4 years ${ }^{(45)}$ or 7 years ${ }^{(46)}$, nor with problem behaviour at 7 years ${ }^{(47)}$. Moreover, $22: 6 n-3$ concentration has been shown to be approximately $32 \%$ lower in umbilical cord blood and $62 \%$ lower in breast milk from vegetarian pregnancies compared with omnivores ${ }^{(48)}$ which suggests that metabolic adaptations that increase $22: 6 n-3$ concentration in maternal blood during pregnancy are insufficient to compensate for low intake of $22: 6 n-3$ in vegetarians ${ }^{(49)}$. One possibility is that maternal adaptations to hepatic PUFA metabolism may be involved in the adjustment of the mother's tissues to pregnancy rather than contributing directly to the PUFA supply and development of the fetus. For example, in the rat increased maternal plasma PC16:0/22: 6 concentration occurs in late pregnancy and may facilitate supply of $22: 6 n-3$ for incorporation into milk $^{(41)}$.

\section{Extra-hepatic PUFA biosynthesis}

Tissues can acquire pre-formed $\geq$ C20 PUFA from blood which are derived from the diet or from hepatic synthesis. These PUFA are assimilated primarily by hydrolysis of TAG and uptake of fatty acids at the surface of the vascular endothelium via the actions of lipoprotein lipase and CD36 or uptake via CD36 from the NEFA pool ${ }^{(50)}$. The Na-dependent transporter Mfsd2a has also been shown to transport lysoPC containing $22: 6 n-3$ or $18: 1 n-9$ selectively to the brain ${ }^{(51,52)}$, skin fibroblasts ${ }^{(53)}$, retina $^{(54)}$ and placenta ${ }^{(55)}$. At least some extra-hepatic tissues express key genes involved in PUFA synthesis or have been shown to convert EFA to longer-chain PUFA. One possible explanation for local intracellular PUFA synthesis is that the pool of pre-formed $\geq 20$ carbon PUFA in blood is insufficient to meet the demands of specific tissues, possibly in terms of quantity, composition and/or timing and that obtaining specific 
PUFA is closely associated with fundamental processes in the function of those cells.

FADS2 mRNA expression has been detected in human heart, brain, lung, liver, skeletal muscle, kidney, pancreas and placenta, while FADS1 was found to be expressed primarily in liver, lung, brain and heart with only trace expression in pancreas, kidney, skeletal muscle and placenta ${ }^{(56)}$. However, the relative expression of FADS2 to other genes involved in the pathway differs between reports ${ }^{(56,57)}$. In general, human brain and liver expressed the highest level of FADS 2 mRNA, followed by heart $>$ placenta $\equiv$ lung $>$ kidney $>$ skeletal muscle $>$ spleen but was essentially undetectable in pancreas ${ }^{(56,57)}$. FADS1, FADS2, ELOVL5 and ELOVL4 have also been shown to be expressed in peripheral blood mononuclear cells (PBMC) ${ }^{(58,59)}$. Moreover, PUFA biosynthesis has been reported in femoral $\operatorname{artery}^{(60)}$, vascular smooth muscle (VSM) cells ${ }^{(61)}$, testis ${ }^{(62)}$, umbilical vein ${ }^{(63)}$, leucocytes ${ }^{(59,64-66)}$ and a number of neoplastic and non-cancerous mammary epithelial cell lines ${ }^{(67,68)}$. There is no evidence that extra-hepatic cells secrete newly synthesised PUFA. Thus, the expression of genes involved in PUFA synthesis and/or PUFA synthesis in extra-hepatic tissues suggests that capacity for EFA interconversion may serve a different purpose to that in the liver. However, relatively few studies have investigated the role of PUFA synthesis in cell function. Nevertheless, emerging findings discussed below suggest that local PUFA synthesis is required for the normal function of at least some cell types.

\section{PUFA biosynthesis in leucocytes}

Leucocytes can convert 18:3n-3 and 18:2n-6 to longer-chain, more unsaturated PUFA, although the extent of such conversion appears to differ between types of immune cells and with the activation state of the cells. Radiolabelled $18: 2 n-6$ can be converted to $20: 2 n-6$ in quiescent murine macrophages without detectable further desaturation or elongation ${ }^{(69)}$. Incubation of murine peritoneal macrophages with the calcium ionophore A23187, phorbol myristate acetate, or zymosan did not induce further interconversion of $20: 2 n-6^{(69)}$. Furthermore, $\left[{ }^{14} \mathrm{C}\right]$ $18: 3 n-6$ can be converted to $20: 3 n-6$, but not to $20: 4 n-6$, in stimulated murine macrophages ${ }^{(66)}$. Absence of detectable $20: 4 n-6$ suggests that murine macrophages lack $\Delta 5$ desaturase activity.

In contrast to macrophages, mitogen stimulation increased the proportions of $18: 1 n-9,22: 5 n-3$ and $22: 6 n-3$ and decreased the proportion of $n-6$ PUFA in T-cell membranes ${ }^{(70)}$. This suggests remodelling of membrane phospholipids and/ or increased synthesis or uptake of specific fatty acids including longer-chain $n$-3 PUFA. Mitogen stimulation of human PBMC increased $\Delta 9, \Delta 6$ and $\Delta 5$ desaturase activities, which was associated with increased interconversion of $\left[{ }^{14} \mathrm{C}\right] 18: 0$ to $18: 1 n-9$ and of $\left[{ }^{14} \mathrm{C}\right] 18: 2 n-6$ and $\left[{ }^{14} \mathrm{C}\right] 18: 3 n-3$ to triene and tetriene PUFA ${ }^{(71)}$. The authors noted that it was uncertain whether such capacity was sufficient to account for the changes in membrane composition that accompany T-cell activation. Moreover, incubation of human PBMC with $18: 2 n-6$ or $18: 3 n-3$ followed by mitogen stimulation increased the incorporation of these fatty acids into the cells ${ }^{(71)}$. Incubation of mitogen-stimulated rat lymph node lymphocytes with $18: 3 n-3$ increased the proportions of $18: 3 n-3,20: 5 n-3$ and $22: 5 n-3$ but not $22: 6 n-3$ in membrane phospholipids ${ }^{(72)}$ which suggests that at least some of the changes in membrane composition in activated $\mathrm{T}$ cells may involve endogenous PUFA synthesis, while other, such as increased 22:6n-3 content, may represent selective uptake from their environment. Although incubation with 18:2n-6 increased the proportion of this fatty acid in T-cell phospholipids, there was only a small, non-significant increase in the $20: 4 n-6$ content $^{(72)}$. The apparent inconsistency in the conversion of $18: 3 n-3$ and $18: 2 n-6$ was not explained by the authors. These findings suggest that, in contrast to macrophages, human and rat lymphocytes express $\Delta 6$ and $\Delta 5$ desaturase, and that elongase expression is up-regulated in activated cells. Increased membrane fluidity induced by increasing the proportion of unsaturated fatty acids in membrane phospholipids has been suggested a possible mechanism by which unsaturated fatty acids could inhibit immune cell function by modifying the membrane protein activity $^{(72)}$. Moreover, increasing the synthesis of PUFA substrates may facilitate the production of pro-resolving mediators and hence attenuate the immune response ${ }^{(73)}$.

A recent study of PUFA biosynthesis in human PBMC confirmed that mitogen stimulation induced increased incorporation of $\left[{ }^{13} \mathrm{C}\right] 18: 3 n-3$ into cell lipids and conversion to longerchain, more unsaturated fatty acids ${ }^{(59)}$. This was accompanied by marked up-regulation of FADS2, FADS1, ELOVL5 and ELOVL4 mRNA expression ${ }^{(59)}$. The first two reactions were chain elongation of $18: 3 n-3$ to $20: 3 n-3$ by an unidentified elongase followed by $\Delta 8$ desaturation of $20: 3 n-3$ to $20: 4 n-3$, essentially the reverse of the initial reactions of the pathway reported in liver ${ }^{(14,34)}$ (Fig. 2). The first $\Delta 6$ desaturation in liver has been shown to be rate limiting ${ }^{(74)}$ and to preferentially catalyse conversion of $18: 3 n-3$ compared with $18: 2 n-6^{(74)}$. Thus, one implication of the apparent substitution of the initial $\Delta 6$ desaturation with elongase activity is that the regulation of the PUFA synthesis pathway in PBMC may differ from that of other tissues. For example, elongases have been shown to differ in substrate preference in terms of chain length and level of unsaturation such that elongase 5 catalyses elongation of 18- and 20-carbon PUFA, while elongase 2 exhibits marked preference of 20- and 22-carbon $\mathrm{PUFA}^{(12)}$. Both of these enzymes preferentially catalyse conversion of $n-3$ compared

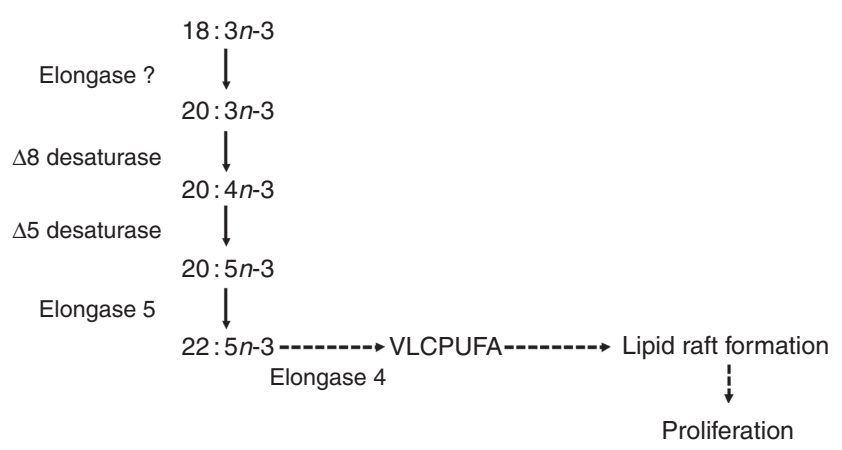

Fig. 2. The proposed pathway for PUFA biosynthesis linked to proliferation of $T$ lymphocytes ${ }^{(57)}$. Dashed arrows indicate putative reactions. VLCPUFA, very long-chain PUFA. 
with equivalent $n$-6 PUFA $^{(12)}$. If so, this may have implications for understanding the influence of dietary fatty acids on immune function. The protein product of the baboon Fads2 gene has been associated with $\Delta 8$ desaturase activity when transfected into yeast cells, although $\Delta 6$ desaturase activity predominated $^{(75)}$. Thus, it is possible that $\Delta 8$ desaturase activity in PBMC was also associated with the protein encoded by the FADS2 gene, although no $\Delta 6$ desaturase activity was detected $^{(59)}$. Moreover, $\Delta 4$-desaturase activity has been demonstrated in MCF7 breast cancer cells transfected with FADS2 that do not normally express this gene ${ }^{(17)}$. Thus, the desaturase activity of the FADS 2 protein product may depend upon the cellular environment in which it is expressed. ELOVL2 was not expressed in PBMC and the PUFA synthesis pathway was truncated after synthesis of $22: 5 n-3^{(59)}$.

ELOVL4 catalyses the conversion of $\geq \mathrm{C} 20$ PUFA, but not $22: 6 n-3$, to PUFA with chain length $\mathrm{C} 24-\mathrm{C} 38^{(76-79)}$, which are involved in the formation and structure of lipid rafts required for T-cell signalling ${ }^{(80)}$. Thus, it has been suggested that truncation of the PUFA synthesis pathway after $22: 5 n-3$ and up-regulation of ELOVL4 may provide very long-chain PUFA required for T-cell activation ${ }^{(59)}$. Moreover, a lipoxygenase-derived metabolite of $20: 3 n-9$ can modulate leukotriene $\mathrm{B}_{4}$ synthesis, and hence neutrophil activation, via inhibition of leukotriene A hydrolase ${ }^{(81)}$. It is not known whether $20: 3 n-3$ can also be converted into bioactive metabolites but demonstration of such activity may suggest an additional mechanism to link $\Delta 8$ desaturation to immune function. Of potential importance to understanding the role of PUFA synthesis in stimulated $\mathrm{T}$ cells, pharmacological inhibition of the FADS2 protein reduced mitogen-induced T-cell proliferation ${ }^{(59)}$. Thus, PUFA synthesis appears to be involved in the regulation of T-cell proliferation possibly by providing substrates for the assembly of lipid microdomains and/or through the formation of novel lipid mediators (Fig. 2).

\section{PUFA synthesis in cancer cells}

PUFA synthesis has been described in several cancer cell types. One recent study compared PUFA synthesis in Jurkat T-cell leukaemia cells with $\mathrm{PBMC}^{(59)}$. The findings showed that interconversion of $\left[{ }^{13} \mathrm{C}\right] 18: 3 n$-3 was constitutive and 17 -fold greater in Jurkat cells than in mitogen-stimulated PBMC. FADS2, FADS1, ELOVL4 and ELOVL5 expression was also up to 17-fold greater in Jurkat cells than in stimulated PBMC, and ELOVL2 was expressed which is consistent with the conversion of 18:3n-3 to $22: 6$ in Jurkat cells. These findings suggest that oncogenic transformation may disrupt PUFA synthesis at the level of transcription at several points in the pathway. The major product of PUFA synthesis in Jurkat cells was $22: 5 n-3$ rather than $20: 3 n-3$, which was the major fatty acid synthesised from 18:3n-3 in PBMC. Jurkat cells expressed both $\Delta 8$ and $\Delta 6$ desaturase activities, while PBMC only expressed $\Delta 8$ desaturase activity. Furthermore, inhibition of the FADS 2 protein did not significantly alter Jurkat cell proliferation ${ }^{(59)}$. Stimulation of PBMC induced hypermethylation of a 600-bp region of the FADS2 promoter. In contrast, this putative regulatory region was hypomethylated in Jurkat cells ${ }^{(59)}$. Together, these findings suggest that PUFA synthesis and its role in regulating lymphocyte cell proliferation is disrupted in Jurkat cells.

The activity of $\Delta 6$ desaturase and the expression of FADS 2 mRNA were shown to be substantially greater in murine xenografts of B16 melanoma and Lewis lung carcinoma (LLC) cells compared with the normal tissue ${ }^{(82)}$. FADS2 RNAi knockdown inhibited proliferation and reduced tumour size in both B16 and LLC tumours ${ }^{(82)}$ which suggests that PUFA synthesis may be involved in the regulation of mitosis in these cells. The ratio of the sum of $n-6$ PUFA to $18: 2 n-6$ in primary breast cancer tissue, a proxy marker of PUFA synthesis, was greater than that in uninvolved tissues ${ }^{(83)}$ and higher in more aggressive oestrogen receptor-negative (ER-) tumours compared with ER+ tumours ${ }^{(83)}$.

The absence of FADS2 expression in MCF7 breast carcinoma cells results in the synthesis by $\Delta 5$ desaturase of $5,11,14-20: 3$ and 5,11,14,17-20:4 which lack the 8-9 double bond of their eicosanoid substrate counterparts $20: 4 n-6$ and $20: 5 n-3^{(84)}$. This suggests a mechanism by which deletion of FADS 2 during malignant transformation may lead to disruption of cellular signalling and the production of novel second messengers ${ }^{(84)}$. Furthermore, activation of the c-Ha-ras oncogene, but not the c-Ha-ras pro-oncogene, in spontaneously immortalised MCF10A mammary epithelial cells resulted in the loss of capacity for $\Delta 6$ and $\Delta 4$ desaturation ${ }^{(85)}$.

Together these studies suggest that altered regulation of PUFA synthesis is a characteristic of at least some types of cancer, which may be linked to their proliferative capacity. This is consistent with the findings of studies that show reduction in proliferation and/or induction of apoptosis of cancer cells treated with $22: 6 n-3^{(86-89)}$.

\section{PUFA synthesis in spermatogenesis}

Human sperm is highly enriched in $22: 6 n-3$. Morphologically normal sperm cells contain approximately $35 \% 22: 6 n-3$, while the proportion of $20: 4 n-6$ is approximately $10 \cdot 5 \%{ }^{(90)}$. The presence of $22: 6 n-3$ in sperm membrane phospholipids has been associated with cell motility, membrane fusion and the synthesis of second messengers and is consistent with the presence of high levels of antioxidant defence mechanisms ${ }^{(90)}$. Experimental EFA deficiency in rats can induce degeneration of seminiferous tubules and reduced sperm formation and fertility $^{(91)}$. Moreover, feeding male Fads2 null mice a diet containing 18:3n-3 and 18:2n-6, without longer-chain PUFA, induced $74 \%$ reduction in the proportion of $22: 6 n-3$ and $92 \%$ decrease in the relative amount of $20: 4 n-6$ (92\%) in testis ${ }^{(92)}$. This was accompanied by arrested spermatogenesis and degradation of seminiferous tissue ${ }^{(92)}$. Dietary supplementation of Fads 2 null mice with $22: 6 n-3$ restored spermatogenesis and fertility to levels equivalent to wild-type mice ${ }^{(92)}$. This suggests that PUFA synthesis is important for fertility in mice fed a diet without longer-chain PUFA. However, because the knockout was not tissue-specific, it is not possible to conclude whether the activity of the PUFA synthesis pathway in testis is involved. However, there is some evidence for local PUFA synthesis in testis. Microsomes from human testis can convert $\left[{ }^{14} \mathrm{C}\right] 18: 2 n-6$ to $20: 3 n-6(88 \%)$ and $20: 4 n-6(12 \%)$ but not $18: 3 n-6^{(62)}$, 
indicating that the initial reactions were elongation of $18: 2 n-6$ to $20: 2 n-6$, followed by $\Delta 8$ desaturation to form $20: 3 n-6$ followed by $\Delta 5$ desaturation to $20: 4 n-6$. This pathway has also been reported in rat testis ${ }^{(93)}$. Moreover, the proportion of 22:6n-3 in testis from humans and from Rhesus macaques increases during puberty and remains essentially unchanged during adulthood ${ }^{(94,95)}$. One possible mechanism is that testicular $22: 6 n-3$ synthesis increases during puberty to meet the requirements for $22: 6 n-3$ during spermatogenesis. If so, this suggests that regulation of PUFA synthesis in testis differs from hepatocytes in which testosterone has no significant effect on $22: 6 n-3$ synthesis $^{(35)}$. However, the contribution of testicular PUFA synthesis to male fertility has yet to be determined.

\section{PUFA synthesis in arteries}

Bovine aortic endothelial cells have been shown to retroconvert $22: 6 n-3$ to $22: 5 n-3$ and $20: 5 n-3^{(96)}$ and $22: 4 n-6$ to $20: 4 n-6^{(97)}$. Elongation of $\left[{ }^{14} \mathrm{C}\right] 20: 5 n-3$ to $22: 5 n-3$ and of $\left[{ }^{14} \mathrm{C}\right] 20: 4 n-6$ to $22: 4 n-6$ has also been shown in human vascular endothelial cells ${ }^{(63)}$, although the role of PUFA synthesis in endothelial cell function has not been reported. Phenylephrine stimulation of isolated rat aortae increased Fads1 and Fads2 mRNA expression compared with unstimulated vessels ${ }^{(60)}$. Moreover, treatment of isolated rat aortae or human femoral artery with the FADS2 protein inhibitor SC26196 or rat aortae with the $\Delta 5$ desaturase inhibitor sesamin reduced phenylephrine-induced vasoconstriction and secretion by rat aortae of pro-constriction eicosanoids $\mathrm{PGF}_{2 \alpha}, \mathrm{PGE}_{2}$ and thromboxane $\mathrm{A} 2^{(60)}$. Thus, PUFA synthesis, which was localised to VSM rather than the endothelium ${ }^{(60)}$, appears to be involved in regulating vasoconstriction. Mouse aortae, immortalised murine VSM (MOVAS) cells and human primary aortic VSM cells express Fads1, Fads2 and Elovl5 but not Elovl $2^{(61)}$. This is consistent with the apparent absence of conversion of $22: 4 n-6$ to $22: 5 n-6$, in MOVAS cells ${ }^{(61)}$. Since Elovl2 was also not expressed in PBMC, it is possible that suppression of Elovl2 transcription is a characteristic of at least some excitable tissues although the precise function may differ. The absence of Elovl2 expression did not appear to involve hypermethylation of the promoter ${ }^{(61)}$. Inhibition of $\Delta 6$ or $\Delta 5$ desaturase significantly reduced phenylephrinemediated $\mathrm{Ca}$ release and secretion of $\mathrm{PGE}_{2}$ and $\mathrm{PGF}_{2 \alpha}$ in MOVAS cells ${ }^{(57)}$. The findings suggest that $\alpha_{1}$-adrenergic receptor-mediated vasoconstriction involves activation of PUFA synthesis acting via regulation of endogenous $\mathrm{Ca}^{2+}$ release and secretion of specific vaso-active eicosanoids. One possible mechanism that has been suggested to explain the link between PUFA synthesis and vasoconstriction is activation of protein kinase $\mathrm{C}_{\zeta}$ and inhibition of myosin light chain phosphorylase by newly synthesised $20: 4 n-6^{(61)}$.

\section{Polymorphisms in genes involved in PUFA biosynthesis}

The region of human chromosome 11 that contains the FADS1 and FADS2 genes in a head-to-head orientation (11q12.2q13.1), together with FADS $3^{(98)}$, contains 4391 variants, of which 217 can result in amino acid changes ${ }^{(99)}$ and hence potentially alter enzyme activity. This region has been identified as a cancer 'hotspot locus' ${ }^{,(84,100)}$. Several genome-wide association studies have reported associations between polymorphisms in genes associated with PUFA synthesis and the concentrations of PUFA in blood. A meta-analysis of 8866 individuals from five cohorts showed that minor alleles of SNP in FADS2 and FADS1 were associated with a higher level of $18: 3 n-3$ and a lower level of $20: 5 n-3,22: 5 n-3$ and $22: 6 n-3$, while minor alleles of ELOVL2 were associated with higher proportions of $20: 5 n-3$ and $22: 5 n-3$ and a lower proportion of $22: 6 n-3^{(101)}$. The variation in the proportions of $n-6$ PUFA in plasma phospholipids explained by eleven locus haplotypes has been shown to be $18: 2 n-6,9 \cdot 2 \% ; 18: 3 n-6,7 \cdot 9 \% ; 20: 2 n-6$, $12 \cdot 3 \% ; 20: 3 n-6,10 \cdot 8 \%$ and $20: 4 n-6,28 \cdot 5 \%{ }^{(97)}$. In contrast, there were only limited associations with $n$-3 PUFA (18:3n-3, $5.4 \% ; \quad 20: 5 n-3, \quad 6.9 \% ; \quad 22: 5 n-3, \quad 5 \cdot 1 \% ; 22: 6 n-3,2 \cdot 9 \%)^{(98)}$. However, whether these polymorphisms alter tissue function is not known. Moreover, interpretation of such associations may be confounded by contributions of hepatic phospholipid biosynthesis by the cytosine diphosphate choline and phosphatidylethanolamine (PE) $N$-methylation pathways to the fatty acid composition of plasma phospholipids because human ${ }^{(102)}$ and rat $^{(41)}$ liver selectively synthesise $20: 4 n$-6- and $22: 6 n$-3-containing PC by PE $N$-methylation. One possible explanation for the greater effect of $F A D S$ polymorphisms on the proportions of $n$-6 PUFA for $n$ - 3 PUFA is the typically greater intake of $18: 2 n-6$ than $18: 3 n-3^{(103)}$ and consequently greater flux of $n-6$ PUFA through the PUFA synthesis pathway than 18:3n-3. Women carrying FADS1 and FADS2 minor alleles had lower proportions of $20: 4 n-6$ and $22: 5 n-3$ in their plasma and in breast milk, although the effect on the development of their infants and children was not assessed ${ }^{(104)}$. Thus, mutations in genes involved in PUFA synthesis are associated with variations in plasma PUFA status, which suggests that hepatic PUFA synthesis can contribute to the levels of circulating PUFA. However, these findings have not been used to estimate the magnitude of this contribution. Carriers of the minor allele (deletion) of a 22 bp FADS 2 insertion-deletion mutation had lower expression of FADS1 in lymphocytes ${ }^{(105)}$ and lower $20: 4 n-6$ status compared with the major insertion allele ${ }^{(106)}$. The frequency of the insertion/insertion allele was greater in populations with a tradition of vegetarian diet compared with Western omnivorous populations who had a higher frequency of the deletion/deletion genotype. This has been suggested as a possible adaption to low intakes of pre-formed longer-chain PUFA ${ }^{(106)}$.

To date, the effect of polymorphisms in genes involved in PUFA synthesis on tissue function has not been investigated directly, although such studies may provide novel insights into tissue function and disease aetiology. However, there is some evidence that individuals carrying minor FADS1-FADS2 alleles that were associated with lower $20: 4 n-6$ status have a lower prevalence of allergic disease which may be attributable to a reduction in the availability of $20: 4 n-6$ for the synthesis of proinflammatory eicosanoids ${ }^{(98)}$. However, since PUFA synthesis can regulate T-cell activation and proliferation ${ }^{(59)}$, it is also possible that reduced desaturase activity may constrain the activity of the PUFA synthesis pathway, thus limiting cell proliferation and the immune response. 


\section{Conclusions}

Humans have limited capacity for hepatic conversion of EFA to longer-chain, more unsaturated fatty acids ${ }^{(18,19)}$. Current knowledge about the contribution of EFA interconversion to meeting demands for PUFA in humans is based primarily on the results of whole-body tracer studies that are difficult to interpret in terms of net contribution of PUFA status and on dietary intervention trials that use amounts of EFA that exceed those found in typical western omnivorous diets ${ }^{(18,19)}$. The relatively few studies that report PUFA status in individuals consuming vegetarian diets that exclude pre-formed $\geq$ C20 PUFA suggest that hepatic PUFA synthesis is unable to supply sufficient longer-chain PUFA to maintain status equivalent to that achieved by consuming an omnivorous diet ${ }^{(49)}$. However, whether the levels of PUFA found in omnivores are optimal or whether they exceed requirements for maintaining health is uncertain. It is possible that humans can function adequately with PUFA status found in vegetarians, which primarily reflects PUFA synthesis from EFA, as may be implied by the health benefits of such diets ${ }^{(49)}$. If so, it may be appropriate to question whether recommendations for intake of $n-3$ PUFA are higher than needed for optimal tissue function as opposed to pharmacological intakes that may prevent specific lifestyleassociated diseases. Overall, the contribution of PUFA synthesis to meeting the demands for $\geq \mathrm{C} 20$ PUFA of individuals is uncertain and requires further consideration.

A number of extra-hepatic tissues express genes involved in PUFA synthesis or have been shown to be capable of converting EFA to longer-chain PUFA. The details of such processes differ between cell types that suggest extra-hepatic PUFA synthesis is involved in and tailored to a particular cell phenotype. However, there are important gaps in knowledge regarding the precise role of PUFA synthesis in each cell type. Nevertheless, the findings of experiments that have attempted to address this problem suggest PUFA synthesis can be involved in key regulatory processes such as cell proliferation and that oncogenic transformation may result in disruption of the link between PUFA synthesis and cell function.

In conclusion, the biological importance of extra-hepatic PUFA biosynthesis remains unclear and there are major gaps in knowledge about PUFA synthesis in humans. Moreover, it may be that the primary role of PUFA biosynthesis within specific tissues lies in local intracellular provision of specific PUFA in a timely manner to meet the demands of specific cell functions.

\section{Acknowledgements}

Thank you to Dr Elizabeth Miles for her helpful comments and suggestions during the preparation of this manuscript.

G. C. B. has received research funding from Nestlé, Abbott Nutrition and Danone. He is a member of the Scientific Advisory Board of BASF and a member of the BASF Asia-Pacific Grant Award Panel. At the time the manuscript was submitted, he was the editor-in-chief of the British Journal of Nutrition but was excluded completely from the peer-review process.

G. C. B. declares no conflicts of interest with the content of this article.

\section{References}

1. Calder PC, Yaqoob P, Harvey DJ, et al. (1994) Incorporation of fatty acids by concanavalin A-stimulated lymphocytes and the effect on fatty acid composition and membrane fluidity. Biochem J 300, 509-518.

2. Wada M, DeLong CJ, Hong YH, et al. (2007) Enzymes and receptors of prostaglandin pathways with arachidonic acidderived versus eicosapentaenoic acid-derived substrates and products. J Biol Chem 282, 22254-22266.

3. Heung YM \& Postle AD (1995) The molecular selectivity of phospholipase D in HL60 granulocytes. FEBS Lett 364, 250-254.

4. Heung YM \& Postle AD (1995) Substrate selectivity of phospholipase D in HL60 granulocytes: effects of fatty acid supplementation. Biochem Soc Trans 23, 276 S.

5. Kamiya Y, Mizuno S, Komenoi S, et al. (2016) Activation of conventional and novel protein kinase $\mathrm{C}$ isozymes by different diacylglycerol molecular species. Biochem Biophys Rep 7, 361-366.

6. Forman BM, Chen J \& Evans RM (1997) Hypolipidemic drugs, polyunsaturated fatty acids, and eicosanoids are ligands for peroxisome proliferator-activated receptors alpha and delta. Proc Natl Acad Sci U S A 94, 4312-4317.

7. Burdge GC \& Lillycrop KA (2014) Fatty acids and epigenetics. Curr Opin Clin Nutr Metab Care 17, 156-161.

8. Ichi I, Kono N, Arita Y, et al. (2014) Identification of genes and pathways involved in the synthesis of Mead acid (20:3n-9), an indicator of essential fatty acid deficiency. Biochim Biophys Acta 1841, 204-213.

9. Barr LH, Dunn GD \& Brennan MF (1981) Essential fatty acid deficiency during total parenteral nutrition. Ann Surg 193, 304-311.

10. Klenk E \& Mohrhauer H (1960) Studies on the metabolism of polyenoic fatty acids in the rat. Hoppe Seylers $Z$ Physiol Chem 320, 218-232.

11. Mead JF (1968) The metabolism of the polyunsaturated fatty acids. Prog Chem Fats Other Lipids 9, 161-192.

12. Gregory MK, Gibson RA, Cook-Johnson RJ, et al. (2011) Elongase reactions as control points in long-chain polyunsaturated fatty acid synthesis. PLOS ONE 6, e29662.

13. Innis SM (1991) Essential fatty acids in growth and development. Prog Lipid Res 30, 39-103.

14. Voss AC \& Sprecher H (1988) Regulation of the metabolism of linoleic acid to arachidonic acid in rat hepatocytes. Lipids 23, 660-665.

15. Voss AC \& Sprecher H (1988) Metabolism of 6,9,12-octadecatrienoic acid and 6,9,12,15-octadecatetraenoic acid by rat hepatocytes. Biochim Biophys Acta 958, 153-162.

16. Voss A, Reinhart M, Sankarappa S, et al. (1991) The metabolism of 7,10,13,16,19-docosapentaenoic acid to $4,7,10,13,16,19$-docosahexaenoic acid in rat liver is independent of a 4-desaturase. J Biol Chem 266, 19995-20000.

17. Park HG, Park WJ, Kothapalli KS, et al. (2015) The fatty acid desaturase 2 (FADS2) gene product catalyzes Delta4 desaturation to yield $n-3$ docosahexaenoic acid and $n-6$ docosapentaenoic acid in human cells. FASEB J 29, 3911-3919.

18. Plourde M \& Cunnane SC (2007) Extremely limited synthesis of long chain polyunsaturates in adults: implications for their dietary essentiality and use as supplements. Appl Physiol Nutr Metabol 32, 619-634.

19. Burdge G (2004) Alpha-linolenic acid metabolism in men and women: nutritional and biological implications. Curr Opin Clin Nutr Metab Care 7, 137-144.

20. Hussein N, Ah-Sing E, Wilkinson P, et al. (2005) Long-chain conversion of $\left[{ }^{13} \mathrm{C}\right]$ linoleic acid and alpha-linolenic acid in response to marked changes in their dietary intake in men. J Lipid Res 46, 269-280. 
21. Emken EA, Adlof RO \& Gulley RM (1994) Dietary linoleic acid influences desaturation and acylation of deuteriumlabeled linoleic and linolenic acids in young adult males. Biochim Biophys Acta 1213, 277-288.

22. Baker EJ, Miles EA, Burdge GC, et al. (2016) Metabolism and functional effects of plant-derived omega-3 fatty acids in humans. Prog Lipid Res 64, 30-56.

23. Burdge GC \& Calder PC (2006) Dietary alpha-linolenic acid and health-related outcomes: a metabolic perspective. Nutr Res Rev 19, 26-52.

24. James MJ, Ursin VM \& Cleland LG (2003) Metabolism of stearidonic acid in human subjects: comparison with the metabolism of other $n-3$ fatty acids. Am J Clin Nutr 77, $1140-1145$.

25. Burdge GC \& Wootton SA (2002) Conversion of alphalinolenic acid to eicosapentaenoic, docosapentaenoic and docosahexaenoic acids in young women. Br J Nutr 88, $411-420$

26. Burdge GC, Jones AE \& Wootton SA (2002) Eicosapentaenoic and docosapentaenoic acids are the principal products of alpha-linolenic acid metabolism in young men. Br J Nutr 88, 355-363.

27. Lohner S, Fekete K, Marosvolgyi T, et al. (2013) Gender differences in the long-chain polyunsaturated fatty acid status: systematic review of 51 publications. Ann Nutr Metab 62, 98-112.

28. Lin YH, Hibbeln JR, Domenichiello AF, et al. (2018) Quantitation of human whole-body synthesis-secretion rates of docosahexaenoic acid and eicosapentaenoate acid from circulating unesterified $\alpha$-linolenic acid at steady state. Lipids 53, 547-558.

29. Burdge GC, Slater-Jefferies JL, Grant RA, et al. (2008) Sex, but not maternal protein or folic acid intake, determines the fatty acid composition of hepatic phospholipids, but not of triacylglycerol, in adult rats. Prostaglandins Leukot Essent Fatty Acids 78, 73-79.

30. Childs CE, Romeu-Nadal M, Burdge GC, et al. (2010) The polyunsaturated fatty acid composition of hepatic and plasma lipids differ by both sex and dietary fat intake in rats. J Nutr 140, 245-250.

31. Isaksson C, Hanson MA \& Burdge GC (2014) The effects of spatial and temporal ecological variation on fatty acid compositions of wild great tits Parus major. J Avian Biol 46, 245-253.

32. Giltay EJ, Gooren LJ, Toorians AW, et al. (2004) Docosahexaenoic acid concentrations are higher in women than in men because of estrogenic effects. Am J Clin Nutr 80, $1167-1174$.

33. Giltay EJ, Duschek EJ, Katan MB, et al. (2004) Raloxifene and hormone replacement therapy increase arachidonic acid and docosahexaenoic acid levels in postmenopausal women. J Endocrinol 182, 399-408.

34. Sibbons CM, Brenna JT, Lawrence P, et al. (2014) Effect of sex hormones on $n-3$ polyunsaturated fatty acid biosynthesis in HepG2 cells and in human primary hepatocytes. Prostaglandins Leukot Essent Fatty Acids 90, 47-54.

35. Childs CE, Kew S, Finnegan YE, et al. (2014) Increased dietary alpha-linolenic acid has sex-specific effects upon eicosapentaenoic acid status in humans: re-examination of data from a randomised, placebo-controlled, parallel study. Nutr J 13, 113.

36. Kuipers RS, Luxwolda MF, Offringa PJ, et al. (2012) Fetal intrauterine whole body linoleic, arachidonic and docosahexaenoic acid contents and accretion rates. Prostaglandins Leukot Essent Fatty Acids 86, 13-20.
37. Reisbick S, Neuringer M, Hasnain R, et al. (1994) Home cage behavior of rhesus monkeys with long-term deficiency of omega-3 fatty acids. Physiol Behav 55, 231-239.

38. Postle AD, Al MD, Burdge GC, et al. (1995) The composition of individual molecular species of plasma phosphatidylcholine in human pregnancy. Early Hum Dev 43, 47-58.

39. Postle AD, Burdge GC \& Al MD (1994) Molecular species composition of plasma phosphatidylcholine in human pregnancy. World Rev Nutr Diet 75, 109-111.

40. Meyer BJ, Onyiaodike CC, Brown EA, et al. (2016) Maternal plasma DHA levels increase prior to 29 days post-LH surge in women undergoing frozen embryo transfer: a prospective, observational study of human pregnancy. J Clin Endocrinol Metab 101, 1745-1753.

41. Burdge GC, Hunt AN \& Postle AD (1994) Mechanisms of hepatic phosphatidylcholine synthesis in adult rat: effects of pregnancy. Biochem J 303, 941-947.

42. Childs CE, Hoile SP, Burdge GC, et al. (2012) Changes in rat $n-3$ and $n-6$ fatty acid composition during pregnancy are associated with progesterone concentrations and hepatic FADS2 expression. Prostaglandins Leukot Essent Fatty Acids 86, 141-147.

43. Crozier SR, Sibbons CM, Fisk HL, et al. (2018) Arachidonic acid and DHA status in pregnant women is not associated with cognitive performance of their children at 4 or 6-7 years. Br J Nutr 119, 1400-1407.

44. Brouwer-Brolsma EM, van de Rest O, Godschalk R, et al. (2017) Associations between maternal long-chain polyunsaturated fatty acid concentrations and child cognition at 7 years of age: the MEFAB birth cohort. Prostaglandins Leukot Essent Fatty Acids 126, 92-97.

45. Ghys A, Bakker E, Hornstra G, et al. (2002) Red blood cell and plasma phospholipid arachidonic and docosahexaenoic acid levels at birth and cognitive development at 4 years of age. Early Human Dev 69, 83-90.

46. Bakker EC, Ghys AJ, Kester AD, et al. (2003) Long-chain polyunsaturated fatty acids at birth and cognitive function at 7 y of age. Eur J Clin Nutr 57, 89-95.

47. Krabbendam L, Bakker E, Hornstra G, et al. (2007) Relationship between DHA status at birth and child problem behaviour at 7 years of age. Prostaglandins Leukot Essent Fatty Acids 76, 29-34.

48. Sanders TA \& Reddy S (1992) The influence of a vegetarian diet on the fatty acid composition of human milk and the essential fatty acid status of the infant. $J$ Pediatr 120, S71-S77.

49. Burdge GC, Tan SY \& Henry CJ (2017) Long-chain $n$-3 PUFA in vegetarian women: a metabolic perspective. J Nutr Sci 6, e58.

50. Goldberg IJ, Eckel RH \& Abumrad NA (2009) Regulation of fatty acid uptake into tissues: lipoprotein lipase- and CD36mediated pathways. J Lipid Res 50, Suppl., S86-S90.

51. Guemez-Gamboa A, Nguyen LN, Yang H, et al. (2015) Inactivating mutations in MFSD2A, required for omega-3 fatty acid transport in brain, cause a lethal microcephaly syndrome. Nat Genet 47, 809-813.

52. Nguyen LN, Ma D, Shui G, et al. (2014) Mfsd2a is a transporter for the essential omega-3 fatty acid docosahexaenoic acid. Nature 509, 503-506.

53. Moore SA, Hurt E, Yoder E, et al. (1995) Docosahexaenoic acid synthesis in human skin fibroblasts involves peroxisomal retroconversion of tetracosahexaenoic acid. J Lipid Res 36, 2433-2443.

54. Wong BH, Chan JP, Cazenave-Gassiot A, et al. (2016) Mfsd2a is a transporter for the essential omega-3 fatty acid docosahexaenoic acid (DHA) in eye and is important for 
photoreceptor cell development. I Biol Chem 291 10501-10514.

55. Prieto-Sanchez MT, Ruiz-Palacios M, Blanco-Carnero JE, et al. (2017) Placental MFSD2a transporter is related to decreased DHA in cord blood of women with treated gestational diabetes. Clinl Nutr 36, 513-521.

56. Cho HP, Nakamura M \& Clarke SD (1999) Cloning, expression, and fatty acid regulation of the human delta- 5 desaturase. J Biol Chem 274, 37335-37339.

57. Cho HP, Nakamura MT \& Clarke SD (1999) Cloning, expression, and nutritional regulation of the mammalian Delta-6 desaturase. I Biol Chem 274, 471-477.

58. Chisaguano AM, Montes R, Perez-Berezo T, et al. (2013) Gene expression of desaturase (FADS1 and FADS2) and elongase (ELOVL5) enzymes in peripheral blood: association with polyunsaturated fatty acid levels and atopic eczema in 4-year-old children. PLOS ONE 8, e78245.

59. Sibbons CM, Irvine NA, Pérez-Mojica JE, et al. (2018) Polyunsaturated fatty acid biosynthesis involving $\Delta 8$ desaturation and differential DNA methylation of FADS2 regulates proliferation of human peripheral blood mononuclear cells. Front Immunol 9, 432.

60. Kelsall CJ, Hoile SP, Irvine NA, et al. (2012) Vascular dysfunction induced in offspring by maternal dietary fat involves altered arterial polyunsaturated fatty acid biosynthesis. PLOS ONE 7, e34492.

61. Irvine NA, Lillycrop KA, Fielding B, et al. (2015) Polyunsaturated fatty acid biosynthesis is involved in phenylephrine-mediated calcium release in vascular smooth muscle cells. Prostaglandins Leukot Essent Fatty Acids $\mathbf{1 0 1}$ 31-39.

62. Albert DH, Rhamy RK \& Coniglio JG (1979) Desaturation of eicosa-11,14-dienoic acid in human testes. Lipids 14, 498-500.

63. Rosenthal MD, Garcia MC, Jones MR, et al. (1991) Retroconversion and delta 4 desaturation of docosatetraenoate $(22: 4(n-6))$ and docosapentaenoate $(22: 5(n-3))$ by human cells in culture. Biochim Biophys Acta 1083, 29-36.

64. Chapkin RS, Miller CC, Somers SD, et al. (1988) Utilization of dihomo-gamma-linolenic acid (8,11,14-eicosatrienoic acid) by murine peritoneal macrophages. Biochim Biophys Acta 959, 322-331.

65. Chapkin RS \& Miller CC (1990) Chain elongation of eicosapentaenoic acid in the macrophage. Biochim Biophys Acta 1042, 265-267.

66. Chapkin RS \& Coble KJ (1991) Utilization of gammalinolenic acid by mouse peritoneal macrophages. Biochim Biophys Acta 1085, 365-370.

67. Grammatikos SI, Subbaiah PV, Victor TA, et al. (1994) Diversity in the ability of cultured cells to elongate and desaturate essential ( $n-6$ and $n$-3) fatty acids. Ann New York Acad Sci 745, 92-105.

68. Grammatikos SI, Subbaiah PV, Victor TA, et al. (1994) n-3 and $n-6$ fatty acid processing and growth effects in neoplastic and non-cancerous human mammary epithelial cell lines. Br J Cancer 70, 219-227.

69. Chapkin RS, Somers SD \& Erickson KL (1988) Inability of murine peritoneal macrophages to convert linoleic acid into arachidonic acid. Evidence of chain elongation. J Immunol 140, 2350-2355.

70. Anel A, Naval J, Gonzalez B, et al. (1990) Fatty acid metabolism in human lymphocytes. I. Time-course changes in fatty acid composition and membrane fluidity during blastic transformation of peripheral blood lymphocytes. Biochim Biophys Acta 1044, 323-331.
71. Anel A, Naval J, Gonzalez B, et al. (1990) Fatty acid metabolism in human lymphocytes. II. Activation of fatty acid desaturase-elongase systems during blastic transformation. Biochim Biophys Acta 1044, 332-339.

72. Calder PC, Yaqoob P, Harvey DJ, et al. (1994) Incorporation of fatty acids by concanavalin A-stimulated lymphocytes and the effect on fatty acid composition and membrane fluidity. Biochem J 300, 509-518.

73. Halade GV, Black LM \& Verma MK (2018) Paradigm shift metabolic transformation of docosahexaenoic and eicosapentaenoic acids to bioactives exemplify the promise of fatty acid drug discovery. Biotechnol Adv 36, 935-953.

74. Rodriguez A, Sarda P, Nessmann C, et al. (1998) Delta6- and delta5-desaturase activities in the human fetal liver: kinetic aspects. J Lipid Res 39, 1825-1832.

75. Park WJ, Kothapalli KS, Lawrence P, et al. (2009) An alternate pathway to long-chain polyunsaturates: the FADS2 gene product delta8-desaturates $20: 2 n-6$ and $20: 3 n-3$. J Lipid Res 50, 1195-1202.

76. Agbaga MP, Brush RS, Mandal MN, et al. (2008) Role of Stargardt-3 macular dystrophy protein (ELOVL4) in the biosynthesis of very long chain fatty acids. Proc Natl Acad Sci U $S$ A 105, 12843-12848.

77. Mandal MN, Ambasudhan R, Wong PW, et al. (2004) Characterization of mouse orthologue of ELOVL4: genomic organization and spatial and temporal expression. Genomics 83, 626-635.

78. Vasireddy V, Uchida Y, Salem N, et al. (2007) Loss of functional ELOVL4 depletes very long-chain fatty acids $(\geq \mathrm{C} 28)$ and the unique omega-O-acylceramides in skin leading to neonatal death. Human Mol Gen 16, 471-482.

79. Suh M \& Clandinin MT (2005) $20: 5 n-3$ but not $22: 6 n-3$ is a preferred substrate for synthesis of $n-3$ very-long- chain fatty acids (C24-C36) in retina. Curr Eye Res 30, 959-968.

80. Iwabuchi K, Nakayama H, Iwahara C, et al. (2010) Significance of glycosphingolipid fatty acid chain length on membrane microdomain-mediated signal transduction. FEBS Lett 584, 1642-1652.

81. Cleland LG, James MJ, Proudman SM, et al. (1994) Inhibition of human neutrophil leukotriene B4 synthesis in essential fatty acid deficiency: role of leukotriene A hydrolase. Lipids 29, 151-155.

82. He C, Qu X, Wan J, et al. (2012) Inhibiting delta-6 desaturase activity suppresses tumor growth in mice. PLOS ONE 7, e47567.

83. Pender-Cudlip MC, Krag KJ, Martini D, et al. (2013) Delta-6desaturase activity and arachidonic acid synthesis are increased in human breast cancer tissue. Cancer Sci 104, 760-764.

84. Park WJ, Kothapalli KS, Lawrence P, et al. (2011) FADS2 function loss at the cancer hotspot 11q13 locus diverts lipid signaling precursor synthesis to unusual eicosanoid fatty acids. PLOS ONE 6, e28186.

85. Grammatikos S, Harvey M, Subbaiah P, et al. (1995) Loss of fatty-acid delta(6) desaturating ability in human mammary epithelial-cells that express an activated C-ha-ras oncogene. Int J Oncol 6, 1039-1046.

86. Liu J \& Ma DW (2014) The role of $n-3$ polyunsaturated fatty acids in the prevention and treatment of breast cancer. Nutrients 6, 5184-5223.

87. Skender B, Vaculova AH \& Hofmanova J (2012) Docosahexaenoic fatty acid (DHA) in the regulation of colon cell growth and cell death: a review. Biomed Pap Med Fac Univ Palacky Olomouc Czech Repub 156, 186-199.

88. Yousefnia S, Momenzadeh S, Seyed Forootan F, et al. (2018) The influence of peroxisome proliferator-activated receptor 
gamma (PPARgamma) ligands on cancer cell tumorigenicity. Gene 649, 14-22.

89. Burdge GC, Rodway H, Kohler JA, et al. (2000) Effect of fatty acid supplementation on growth and differentiation of human IMR-32 neuroblastoma cells in vitro. J Cell Biochem 80, 266-273.

90. Lenzi A, Picardo M, Gandini L, et al. (1996) Lipids of the sperm plasma membrane: from polyunsaturated fatty acids considered as markers of sperm function to possible scavenger therapy. Human Reprod Update 2, 246-256.

91. Leath WMF, Northop CA, Harrison FA, et al. (1983) Effect of linoleic acid and linolenic acid on testicular developments in the rat. QJ Exp Physiol 68, 221-231.

92. Roqueta-Rivera M, Stroud CK, Haschek WM, et al. (2010) Docosahexaenoic acid supplementation fully restores fertility and spermatogenesis in male delta-6 desaturasenull mice. J Lipid Res 51, 360-367.

93. Albert DH \& Coniglio JG (1977) Metabolism of eicosa-11,14dienoic acid in rat testes. Evidence for delta8-desaturase activity. Biochim Biophys Acta 489, 390-396.

94. Coniglio JG, Grogan WM Jr \& Rhamy RK (1975) Lipid and fatty acid composition of human testes removed at autopsy. Biol Reprod 12, 255-259.

95. Connor WE, Lin DS \& Neuringer M (1997) Biochemical markers for puberty in the monkey testis: desmosterol and docosahexaenoic acid. J Clin Endocrinol Metab 82, 1911-1916.

96. Hadjiagapiou C \& Spector AA (1987) Docosahexaenoic acid metabolism and effect on prostacyclin production in endothelial cells. Arch Biochem Biophys 253, 1-12.

97. Mann CJ, Kaduce TL, Figard PH, et al. (1986) Docosatetraenoic acid in endothelial cells: formation, retroconversion to arachidonic acid, and effect on prostacyclin production. Arch Biochem Biophys 244, 813-823.

98. Schaeffer L, Gohlke H, Muller M, et al. (2006) Common genetic variants of the FADS1 FADS2 gene cluster and their reconstructed haplotypes are associated with the fatty acid composition in phospholipids. Human Mol Gene $\mathbf{1 5}$, 1745-1756.

99. Minihane AM (2016) Impact of genotype on EPA and DHA status and responsiveness to increased intakes. Nutrients $\mathbf{8}, 123$.

100. Marquardt A, Stohr H, White K, et al. (2000) cDNA cloning, genomic structure, and chromosomal localization of three members of the human fatty acid desaturase family. Genomics 66, 175-183.

101. Lemaitre RN, Tanaka T, Tang W, et al. (2011) Genetic loci associated with plasma phospholipid $n-3$ fatty acids: a metaanalysis of genome-wide association studies from the CHARGE Consortium. PLoS Genetics 7, e1002193.

102. Pynn CJ, Henderson NG, Clark H, et al. (2011) Specificity and rate of human and mouse liver and plasma phosphatidylcholine synthesis analyzed in vivo. J Lipid Res $\mathbf{5 2}$, 399-407.

103. Burdge GC \& Calder PC (2005) Conversion of alpha-linolenic acid to longer-chain polyunsaturated fatty acids in human adults. Reprod Nutr Dev 45, 581-597.

104. Xie L \& Innis SM (2008) Genetic variants of the FADS1 FADS2 gene cluster are associated with altered $(n-6)$ and $(n-3)$ essential fatty acids in plasma and erythrocyte phospholipids in women during pregnancy and in breast milk during lactation. J Nutr 138, 2222-2228.

105. Reardon HT, Zhang J, Kothapalli KS, et al. (2012) Insertiondeletions in a FADS2 intron 1 conserved regulatory locus control expression of fatty acid desaturases 1 and 2 and modulate response to simvastatin. Prostaglandins Leukotrienes Essent Fatty Acids 87, 25-33.

106. Kothapalli KS, Ye K, Gadgil MS, et al. (2016) Positive selection on a regulatory insertion-deletion polymorphism in FADS2 influences apparent endogenous synthesis of arachidonic acid. Mol Biol Evol 33, 1726-1739. 